(interburst interval) and relative power of delta EEG frequency band values surrounding the point $\mathrm{pCO}_{2}$ measurements were averaged using a specified smoothing window.

Results It is shown that by combining the measurements of both a defined period of EEG interburst interval and the relative power of delta EEG frequency band using a multivariate linear regression model, a prediction of $\mathrm{pCO}_{2}$ can be performed. The automatic removal of mechanical artefact and artefact due to other external influences is demonstrated. A regression coefficient $\left(R^{2}\right)$ of 0.64 is obtainable using both the interburst and delta relative power as predictors for $\mathrm{pCO}_{2}$. All variables are significant to within $\mathrm{p}<0.05$. A section of continuous prediction of $\mathrm{pCO}_{2}$ using EEG showing correlation with simultaneous transcutaneous carbon dioxide measurement is demonstrated.

Conclusion The ability to provide a novel non-invasive continuous monitoring of $\mathrm{pCO}_{2}$ in newborn preterm babies is discussed.

\section{AN EVALUATION OF THE USE OF ENTERAL NUTRITION DURING HYPOTHERMIA TREATMENT FOR PERINATAL HYPOXIC ISCHAEMIC ENCEPHALOPATHY}

doi:10.1136/archdischild-2012-302724.1108

'E Tillqvist, ${ }^{2 B}$ Thyagarajan, ${ }^{2}$ V Baral, 'B Hallberg, ${ }^{3} \mathrm{~B}$ Vollmer, 'M Blennow. ' Karolinska University Hospital, Karolinska Institutet, Stockholm, Sweden; 'Neonatal Medicine, Princess Anne Hospital; ${ }^{3}$ Paediatric Neurology, University of Southampton, Southampton, UK

Background There is widespread variation in enteral feeding practices of infants receiving therapeutic hypothermia (cooling) following hypoxic ischemic encephalopathy (HIE). We compared the safety and efficacy of early versus delayed enteral feeding during cooling

Methods Retrospective case control study (January 2009 - December 2011). Cooled infants at Karolinska Hospital, Stockholm (KH) received early enteral feeding and were compared to similar infants at Princess Anne Hospital (PAH) Southampton, who had delayed feeding (controls). Infants also received early parenteral nutrition in both centres.

Results A complete data set was available for 28/37 infants at $\mathrm{PAH}$ compared to $51 / 51$ neonates at KI. Mean baseline parameters at $\mathrm{PAH} / \mathrm{KH}$ were birth weight (3404.80/3723 g), male/female ratio $(50 / 55 \%)$, umbilical arterial $\mathrm{pH}(7.1 / 7.04)$ and base deficit $(-15.65 /-12.03)$

There were differences in enteral feeding rates at $\mathrm{PAH} / \mathrm{KH}$ $(20.1 / 91.0 \%)$. The mean volume of enteral feeds (mls $/ \mathrm{kg} /$ day) at $\mathrm{PAH} / \mathrm{KH}$ on days $1-4$ were: $0 / 2.1,0.2 / 6.1,1.8 / 10.1,1.9 / 17.1$

There were also differences $(\mathrm{PAH} / \mathrm{KH})$ in mean time to establishing full nasogastric tube feeding (5.9/7.2 days) achieving full oral feeds (7.45/10.1 days) and breast feeding rates at discharge $(56 / 70.2 \%)$. The mean length of stay was $9.77 / 14.7$ days $(\mathrm{PAH} / \mathrm{KH})$.

One baby developed spontaneous intestinal perforation at $\mathrm{KH}$ but none developed necrotising enterocolitis in either centres.

Conclusion Feeding practices during hypothermia varies between centres. Early enteral feeding during hypothermia is safe and not associated with any additional morbidity. However, delayed introduction of enteral feeds does not delay the time to reach full enteral feeds or prolong the length of stay at hospital.

\section{TOTAL BODY HYPOTHERMIA AND CIRCULATING BIOMARKERS OF LIVER FUNCTION}

doi:10.1136/archdischild-2012-302724.1109

'D Gardner, ${ }^{2} \mathrm{~S}$ Sanka, ${ }^{1} \mathrm{~A}$ Pawaletz, ${ }^{2} \mathrm{H}$ Muniraman, ${ }^{3} \mathrm{C}$ Jennings, ${ }^{3} \mathrm{~A}$ Vayalakkad, ${ }^{3,4}$ S Victor, ${ }^{2} \mathrm{P}$ Clarke, ${ }^{1,5} \mathrm{MA}$ Turner. 'Neonatal Unit, Liverpool Women's NHS Foundation Trust, Liverpool; ' ${ }^{2}$ Neonatal Unit, Norfolk and Norwich University University Hospitals NHS Foundation Trust, Norwich; ${ }^{3}$ Newborn Intensive Care Unit, Central Manchester University Hospitals NHS Foundation Trust; ${ }^{4}$ Developmental Biomedicine Research
Group, University of Manchester, Manchester; 'Women's and Chidren's Health University of Liverpool, Liverpool, UK

Background and Aims Total body hypothermia (cooling) improves outcome in hypoxic-ischaemic encephalopathy (HIE). This study tested the hypothesis that cooling affects the liver by examining whether cooling during HIE was associated with differences in clinically relevant biomarkers of hepatic metabolism.

Methods Clinical records in 3 centres were searched for babies with HIE and umbilical artery $\mathrm{pH}$ at birth $\leq 7.0$ born between 01/07/2006 and 30/06/2011. Each centre adopted routine cooling on a different date. The results of blood tests reflecting hepatic metabolism measured according to clinical practice within 7 days of birth were collected. ANOVA was used to assess the associations between extreme values of each analyte, HIE grade and the use of cooling and to calculate estimated marginal means for each condition.

Results 127 babies were identified including 31 with Grade 1 (42\% cooled), 65 with Grade 2 (80\% cooled) and 31 babies with Grade 3 ( $90 \%$ cooled). Grade of HIE was associated with maximum AST [HIE1: mean 180 (s.e. 120); HIE2: 367 (85); HIE3: 850, (123)], maximum prothrombin time [HIE1: 18 (3); HIE2: 22 (2); HIE3: 36 (4)] maximum bilirubin [HIE1: 117 (9); HIE2: 108 (8); HIE3 68 (15)] and minimum albumin [HIE1: 28.5 (0.9); HIE2: 23.6 (0.7); HIE3: 20.1 (1)] but not with maximum ALT or maximum APTT. Cooling was not associated with any variables.

Discussion Clinically graded HIE was associated with markers of liver function. Cooling did not modify these associations. Liver and brain may have different susceptibilities to hypoxic-ischaemia or different responses to cooling

\section{IMPLEMENTING A THERAPEUTIC HYPOTHERMIA PROGRAM FOR THE TREATMENT OF PERINATAL HYPOXIC ENCEPHALOPATHY: EXPERIENCE FROM A UK TERTIARY NEONATAL CENTRE}

doi:10.1136/archdischild-2012-302724.1110

K Povazai, E Molnar, M Chilton, A Kapetanakis. Neonatal Medicine, Evelina Children's Hospital Guy's and St Thomas' Hospital NHS Foundation Trust, Kings Health Partners, London, UK

Introduction Hypoxic-ischaemic encephalopathy (HIE) is a leading cause of neonatal morbidity and mortality. Therapeutic hypothermia $(\mathrm{TH})$ is an effective neuroprotective treatment. In the U.K NICE endorsed selected TH use in 5/2010. Access to treatment is increasing. Our institution is a tertiary neonatal unit serving SouthEast London perinatal network. We designed and implemented a TH program with established clinical governance procedures and a rolling training program followed by the treatment of the first patient in 8/2009.

Aim To present our experience in implementation of our $\mathrm{TH}$ program.

Methods Review of clinical records, aEEG, EEG and MRI of the infants treated with TH from 8/2009-3/2012.

Results 44 infants with moderate or severe HIE were treated. Mean GA:40 weeks (36-42 weeks). 61\% outborn (N=27) 10/27 from outside SE-London. Treatment commenced at median age:2.5h for inborns. Outborns commenced passive cooling. On admission 30\% of the outborns had rectal temperature $<30^{\circ} \mathrm{C} 48 \%$ of patients were treated for seizures before $\mathrm{TH}, 16 \%$. During treatment 1 infant developed arrhythmia (PEA) after phenytoin. One infant required extended TH for seizures. $77 \%$ survived to discharge.

93\% had brainMRI performed.

Conclusions Successful introduction of a TH program requires an on-going education program and established clinical governance procedures. Access toTH and transport procedures should be further improved. TH should ideally be provided in centres equipped to provide neurocritical intensive care able to address the complex 
medical needs of patients and families. There is urgent need to refine current $\mathrm{TH}$ protocols and encourage clinical trials of additional neuroprotective treatments.

\section{PILOT CASE SERIES ON THE USE OF MAGNESIUM SULPHATE AS NEUROPROTECTION IN HIE BABIES IN THE COOLING ERA}

doi:10.1136/archdischild-2012-302724.1111

WB Poon, SKY Ho. Neonatal and Developmental Medicine, Singapore General Hospital, Singapore, Singapore

Background and Aims A recent RCT suggested improved neurological outcome at discharge for moderate to severe perinatal asphyxia babies given iv magnesium sulphate. However, this trial was performed in babies who were not cooled.

Methods We present a pilot case series of 3 patients with moderate to severe HIE who satisfied the criteria for cooling and received both cooling and iv magnesium sulphate loading of $200 \mathrm{mg} / \mathrm{kg}$. Serum Magnesium levels were monitored at 0, 12, 24, 48, 72 hours of cooling.

The babies were reviewed for adverse effects of magnesium sulphate in terms of hypotension, arrhythmia, feed intolerance, respiratory depression and hypocalcemia.

Results One patient received systemic cooling and two other patients received selective head cooling. In addition to iv magnesium sulphate loading, decision was made to institute continuous infusion of iv magnesium sulphate in one of these patients for 4 days at $20-40 \mathrm{mg} / \mathrm{kg} / \mathrm{h}$ for PPHN. All babies achieved serum magnesium levels of $>1.2 \mathrm{mmol} / \mathrm{l}$ within $24 \mathrm{~h}$ of the loading dose, which was similar to the level aimed for in the previous RCT.

Magnesium sulphate was well tolerated with only mild hypotension requiring one day of dopamine $(\max 5 \mathrm{mcg} / \mathrm{kg} / \mathrm{min})$ in one patient. No babies had respiratory depression, arrhythmia, feed intolerance or hypocalcemia. Neurodevelopmental outcome to date is also presented.

Conclusions Magnesium sulphate is well tolerated in babies with moderate to severe HIE in the cooling era. A large RCT is required to assess its efficacy, long term impact and further look into adverse effects.

\section{2 \\ A TERTIARY NEONATAL UNIT'S EXPERIENCES IN ESTABLISHING AN IN-HOUSE COOLING SERVICE}

doi:10.1136/archdischild-2012-302724.1112

${ }^{1} \mathrm{R}$ Broughton, ${ }^{2} \mathrm{~V}$ Shaw, ${ }^{2} \mathrm{M}$ Borooah, ${ }^{3} \mathrm{SV}$ Rasiah. ${ }^{2}$ University of Birmingham; ${ }^{2}$ Neonatal Intensive Care Unit, Birmingham Women's Hospital NHS Foundation Trust; ${ }^{3}$ Neonatal Intensive Care Unit, Birmingham Women's Hospital NHS Foundation Trust, Birmingham, UK

Background Hypoxic Ischaemic Encepalopathy (HIE) affects 1-2 per 1,000 live births in UK. The TOBY study showed that therapeutic hypothermia $(\mathrm{TH})$ is beneficial for babies with moderate HIE. In view of this we established an in-house cooling service.

Aim To review our experiences in establishing an in-house cooling service.

Methods The Badger electronic database was used to identify babies who received TH in the last 2 years $(01 / 01 / 10$ to 31/12/11). The management and outcomes were analysed.

Results In the last two year, 27 babies' commenced in-house TH. 3 babies were transferred out to another unit for TH for bed capacity reasons. A further 3 babies died before discharge home. 19 babies received the full 72 hours of cooling in our centre. We did not encounter any major complications with the servo-controlled cooling mattress. Only $50 \%$ of babies had their MRI in the defined time period as per the TOBY guidelines. All babies are being followed up by a dedicated consultant Neonatologist and neurodevelopmental physiotherapist to assess their neurodevelopment up to the age of 2 years.

Conclusion We have safely established an in-house cooling service by following the TOBY guidelines. The servo-controlled cooling mattress provides a safe cooling process with a rectal probe. Identifying these babies early and the interpretation of CFAM was an important aspect of training. Our main challenge was to get an MRI post cooling in a timely fashion. This has been resolved with an agreed dedicated slot for these babies at Birmingham Children's Hospital.

\section{3D DIGITAL CAPTURE OF HEAD CIRCUMFERENCE AND VOLUME IN NEONATES - A METHOD EVALUATION}

doi:10.1136/archdischild-2012-302724.1113

S Ifflaender, M Rüdiger, A Koch, W Burkhardt. Department of Neonatology and Paediatric Intensive Care, University Hospital Carl Gustav Carus, Dresden, Germany

Background Manual measurement of head circumference (HC) is used to quantify head growth in preterm infants. Laser shape digitizers offer semi-automatic HC measuring and additional information on head volume (HV). Reliability and accuracy in obtaining $\mathrm{HC}$ and $\mathrm{HV}$ in neonates has not been investigated yet.

\section{Aims}

1. To determine intraobserver and interobserver variability of $\mathrm{HC}$ and $\mathrm{HV}$ measurements in neonates with a $3 \mathrm{D}$ digital capture system.

2. To compare the method with manual HC measurements

Methods Standard weekly HC measurements on a neonatal unit were conducted manually and digitally with STARScanner laser shape digitizer (Vorum Research Corp., Vancouver, BC) over 12 months. Method comparison was performed using Passing-Bablok-Regression (PBR), Cusum test and Bland-Altman (BA) analyses. Multiple scan examinations by different trained observers were performed to obtain intraobserver/interobserver data.

\section{Results}

1. Intraobserver coefficient of variation was low for $\mathrm{HC}$ (0.1$0.9 \%)$ and $\mathrm{HV}(0.54-1.1 \%)$. BA (mean percentage of difference $\left.M_{d^{\prime}} 95 \% \mathrm{CI}\right)$ of interobserver data showed interchangeability for HC $\left(M_{d}-0.005 ; C I-0.39-0.39\right)$ and $\mathrm{HV}\left(M_{d} 1.51 ; C I\right.$ $-1.17-4.1)$.

2. Method comparison data was acquired from 446 measurements in 258 infants (HC $318 \pm 19.5 \mathrm{~mm}$ ). Overall agreement was good $\left(M_{d}-0.82 ; C I-4.89-3.24\right)$, PBR showed no significant systematic or proportional differences $(a=1.03, \mathrm{CI}$ 0.99-1.06; $\beta=-7.06 C I-17.7-3.01)$. There was no significant deviation from linearity $(p=0.62)$

Conclusions Infant head shape capturing with the examined device is reliable, accurate and save. It offers additional information on HV. Possible benefits of HV in quantifying head growth in preterm infants need to be further investigated.

\section{RESUSCITATION WITH HYDROGEN GAS MIXED INTO SYNTHETIC AIR REDUCES APOPTOSIS IN NEONATAL HYPOXIA-ISCHEMIA PIGLET MODEL}

doi:10.1136/archdischild-2012-302724.1114

R Solberg, L Pankratov, OD Saugstad. Department of Paediatric Research, Os/o University Hospital - Rikshospitalet, Oslo, Norway

Background and Aims Asphyxia and subsequent reoxygenation cause a burst of oxygen free radicals. A gas mixture of ambient air and hydrogen may provide early neuroprotection.

Hydrogen may act as a therapeutic antioxidant by selectively reducing cytotoxic oxygen radicals and thereby contribute to less apoptosis. 